\title{
Assessing the impact of chemotherapy-induced peripheral neurotoxicity on the quality of life of cancer patients
}

\author{
The introduction of a new measure
}

\author{
C. M. L. Driessen • K. M. E. de Kleine-Bolt • \\ A. J. J. M. Vingerhoets • F. Mols • G. Vreugdenhil
}

Received: 31 January 2011 / Accepted: 28 November 2011 /Published online: 9 December 2011

(C) The Author(s) 2011. This article is published with open access at Springerlink.com

\begin{abstract}
Purpose To investigate the impact of chemotherapy-induced neurotoxicity on daily activities and quality of life (QoL) of cancer patients.

Methods QoL of all patients visiting the oncological outpatient ward of the Maxima Medical Centre in the Netherlands from October 2006 until March 2007 treated with taxanes, vinca-alkaloids and/or platinum compounds $(n=88)$ was compared with the QoL of patients that did not receive these treatments yet $(n=43)$. Patient-reported neuropathy symptoms were evaluated with the newly developed Chemotherapy Induced Neurotoxicity Questionnaire (CINQ) and the Functional Assessment of Cancer Therapy/Gynaecologic Oncology Group/Neurotoxicity (FACT/GOG-Ntx) questionnaire.

Results Patients treated with chemotherapy reported significantly more complaints of neuropathy $(p<0.001)$ and more paresthesias and dysesthesias in the upper $(p<0.001 ; p<0.01)$ and lower extremities $(p<0.001)$ compared to those not treated with chemotherapy. They additionally experienced problems with fine motor function (e.g., getting (un)dressed, writing, and picking up small objects). Moreover, cold-induced paresthesias were frequently
\end{abstract}

C. M. L. Driessen · K. M. E. de Kleine-Bolt • G. Vreugdenhil Department of Internal Medicine, Maxima Medical Centre, Veldhoven, The Netherlands

A. J. J. M. Vingerhoets

Clinical Psychology Section, Tilburg University,

Tilburg, The Netherlands

F. Mols $(\bowtie)$

Center of Research on Psychology in Somatic Diseases (CoRPS),

Department of Medical Psychology, Tilburg University,

PO Box 90153, 5000 LE Tilburg, The Netherlands

e-mail: F.mols@Tilburguniversity.edu reported. Overall, patients indicated that their neuropathy had a negative effect on QoL.

Conclusions The newly developed CINQ and the FACT/ GOG-Ntx results suggest a considerable negative impact of patient-reported neuropathy symptoms on daily activities and QoL in cancer patients treated with chemotherapy. However, further validation of the CINQ is needed.

Keywords Cancer - Oncology Neurotoxicity . Chemotherapy $\cdot$ Quality of life

\section{Introduction}

Chemotherapy, the major treatment modality in oncology, is often accompanied by side effects, including peripheral neuropathy. This may have a great impact on quality of life (QoL) [1-5]. Peripheral neurotoxicity is caused by chemotherapeutic agents, like vinca-alkaloids, platinum compounds, and taxanes [6]. The degree of neurotoxicity depends on the type and combination of drugs used, the duration of administration, and the cumulative dose applied $[7,8]$. Depending on the drug, sensory, painful neuropathies (cisplatin, oxaliplatin and carboplatin) or combined sensomotory neuropathies (vincristin and paclitaxel) may occur $[2,8,9]$. In some cases, these symptoms first emerge or worsen after cessation of chemotherapy, in particular in the case of treatment with cisplatin $[8,10]$.

Attempts to evaluate the treatment of peripheral neurotoxicity have yielded promising, but variable results $[6,9-$ 14]. None of the known neuroprotective agents is currently recommended as a standard treatment $[6,11,15]$. Currently, peripheral neuropathy is treated symptomatically. Dose 
reduction or lowering dose intensities are typical ways to prevent peripheral neuropathy, but this may also affect the therapeutic potential.

Until now, there have been few systematic assessments of the impact of peripheral neuropathy problems, which may partly be explained by the lack of adequate measures. Special neurotoxicity supplements have been added to QOL measures, such as the FACT-taxane [1], the Functional Assessment of Cancer Therapy/Gynaecologic Oncology Group-Neurotoxicity (FACT/GOG-Ntx) [4], and the Chemotherapy Induced Peripheral neuropathy (CHIP) [5, 7]. However, these measures fail to evaluate the effects of neurotoxicity on daily functioning.

The aim of this study was to investigate the consequences of patient-reported chemotherapy-induced neuropathy on QoL with a newly developed Chemotherapy Induced Neurotoxicity Questionnaire (CINQ). The FACT/GOG-Ntx was used to validate the CINQ.

\section{Patients and methods}

Patients

All patients visiting the outpatient ward of the Department of Oncology at the Maxima Medical Centre in the Netherlands, between October 2006 and March 2007, who were treated with taxanes, vinca-alkaloids and/or platinum compounds or discontinued treatment less than a month ago were considered eligible. Patients with other comorbid conditions potentially causing CIN such as diabetes, thyroid disease or preexisting neuropathy were excluded. Patients gave informed consent before entering the study.

We compared patients prior to initiation of chemotherapy $(\mathrm{CT}-; n=43)$ with patients who had received at least three cycles with taxanes, vinca-alkaloids or platinum compounds $(\mathrm{CT}+; n=88)$. In this group, data were collected within 1 month after cessation of therapy. The median number of chemotherapy cycles per patient was 6 , ranging from 3 to 14.

\section{Measures}

The newly developed CINQ was based on the questionnaire by Leonard et al. [16] and interviews with seven patients treated with vinca-alkaloids, platinum compounds or taxanes, specifically asking about neurotoxicity symptoms, and their impact on daily functioning. The preliminary CINQ was evaluated by experts and was subsequently adjusted.

The final version consists of experienced symptoms: (A) in arms, hands, fingers; (B) legs, feet, toes; (C) face and mouth, (D) other symptoms, and how this affects QoL. Each symptom question is followed by a question on the amount of experienced bother and the influence on daily activities. For example, "Do you experience cramps in your hands (Yes/No)?" If yes; "How much did this bother you (range $0-5)$ ?" and "Did this influence your daily activities (range $0-5)$ ?" A high score indicates more severe patient-reported neurotoxicity symptoms.

To assess the validity of the Dutch CINQ, the Dutch version of the FACT/GOG questionnaire [2, 3] was used, which can be used for patients with any form of malignancy. This questionnaire contains 27 items, divided into four areas of QoL: (1) physical well-being, (2) social/family wellbeing, (3) emotional well-being and (4) functional wellbeing. The validated Ntx extension of the FACT/GOG questionnaire contains 11 questions which assess chemotherapy-induced peripheral neuropathy [17]. A lower score on the FACT/GOG indicates lower QoL. In addition, a lower score on the NTX component means that patients experience a greater impact of neuropathic symptoms in their life.

\section{Statistical analyses}

We compared socio-demographic and clinical characteristics of the $\mathrm{CT}+$ and $\mathrm{CT}-$ group, using $t$-tests for continuous variables and chi-square analyses for categorical variables (Table 1). The Mann-Whitney test was used to evaluate the differences in neuropathy between these groups (Table 2). The association between the measures was computed with the Pearson $r$ correlation. Statistical test were two-sided and significant if $p<0.05$. All analyses were conducted using SPSS version 17.0.

\section{Results}

\section{Demographics}

Of the total group of 146 eligible patients, three did not meet our inclusion criteria (brain metastases, a transverse lesion, and no signed informed consent form). The sociodemographic and clinical characteristics of the remaining 143 patients (88 CT+; 43 CT-) are compared in Table 1 . The groups did not differ significantly in terms of mean age, sex and indication of treatment.

Impact of neurotoxicity on quality of life

$\mathrm{CT}+$ patients reported significantly more complaints of neuropathy compared to $\mathrm{CT}-$ patients $(110$ vs. $124 ; p<0.001)$ as measured by the FACT/GOG. They also scored significantly lower on the FACT/GOG-Ntx (33 vs. $41 ; p<0.001$ ). Finally, the $\mathrm{CT}+$ patients obtained significantly higher 
Table 1 Socio-demographic and clinical characteristics of patients treated with chemotherapy $(\mathrm{CT}+)$ and a control group (CT-)

$n s$ Not significant

${ }^{\mathrm{a}} \mathrm{CT}-$ : control group of patients before the start of their treatment with chemotherapy

${ }^{\mathrm{b}} \mathrm{CT}+$ : patients with at least three cycles of chemotherapy

'The CT- patients' column represents planned chemotherapy regimens; the $\mathrm{CT}+$ column represents patients that already underwent at least three cycles of chemotherapy

Table 2 Mean scores on specific CINQ questions and results of Mann-Whitney test

Paresthesia is a sensation of tingling, pricking, or numbness of a person's skin

Dysesthesia is an unpleasant, abnormal sense of touch

\begin{tabular}{|c|c|c|c|}
\hline & \multicolumn{3}{|l|}{$\mathrm{N}(\%)$} \\
\hline & Group $\mathrm{CT}^{-}{ }^{\mathrm{a}}(n=43)$ & Group $\mathrm{CT}+{ }^{\mathrm{b}}(n=88)$ & $p$ Value \\
\hline \multicolumn{4}{|l|}{ Socio-demographics } \\
\hline Age (mean (min/max) SD) & $61(36 / 79) 11.4$ & $62(36 / 81) 10.0$ & ns \\
\hline Gender & & & ns \\
\hline Male & $19(44)$ & $39(44)$ & \\
\hline Female & $24(56)$ & $49(56)$ & \\
\hline Indication & & & ns \\
\hline Neo-adjuvant & $1(2)$ & $4(5)$ & \\
\hline Adjuvant & $17(40)$ & $36(41)$ & \\
\hline Curative & $2(5)$ & $7(8)$ & \\
\hline Palliative & $23(53)$ & $41(47)$ & \\
\hline Type of malignancy & & & ns \\
\hline Breast & $7(16)$ & $13(15)$ & \\
\hline Colon & $16(37)$ & $29(33)$ & \\
\hline Sigmoïd & $3(7)$ & $9(10)$ & \\
\hline Rectal & $9(21)$ & $7(8)$ & \\
\hline Gastric & 0 & $6(7)$ & \\
\hline Non Hodgkin lymphoma & $1(2)$ & $8(9)$ & \\
\hline Prostate & 0 & $7(8)$ & \\
\hline Ovarian & $2(5)$ & $5(6)$ & \\
\hline Pseudo myxoma peritonei & $1(2)$ & 0 & \\
\hline Lung & $0(0)$ & $1(1)$ & \\
\hline Endometrial & $0(0)$ & $2(2)$ & \\
\hline Larynx & $1(2)$ & 0 & \\
\hline Multiple myeloma & $1(2)$ & 0 & \\
\hline Hodgkin lymphoma & $1(2)$ & 0 & \\
\hline Esophagus & 0 & $1(1)$ & \\
\hline Pancreatic & $1(2)$ & 0 & \\
\hline Chemotherapy $^{\mathrm{c}}$ & & & ns \\
\hline Oxaliplatin & $29(67)$ & $45(51)$ & \\
\hline Paclitaxel & $9(21)$ & $15(17)$ & \\
\hline Docetaxel & 0 & $12(14)$ & \\
\hline Carboplatin & 0 & $1(1)$ & \\
\hline Cisplatin & $2(5)$ & $8(9)$ & \\
\hline Vincristin & $2(5)$ & $7(8)$ & \\
\hline Vinblastin & $1(2)$ & 0 & \\
\hline
\end{tabular}

\begin{tabular}{lccc}
\hline & \multicolumn{2}{l}{ Mean score \pm SD (range) } & \\
\cline { 2 - 4 } & Group CT- $(n=43)$ & Group CT $+(n=88)$ & $p$ Value \\
\hline Paresthesia upper extremity & $0.2 \pm 0.5(0-2)$ & $1.9 \pm 1.8(0-5)$ & $<0.001$ \\
Dysesthesia upper extremity & $0.2 \pm 0.7(0-3)$ & $0.8 \pm 1.3(0-5)$ & $<0.01$ \\
Paresthesia lower extremity & $0.3 \pm 0.8(0-3)$ & $1.5 \pm 1.7(0-5)$ & $<0.001$ \\
Dysesthesia lower extremity & $0.2 \pm 0.8(0-4)$ & $1.1 \pm 1.6(0-5)$ & $<0.001$ \\
Paresthesia mouth & $0.2 \pm 0.7(0-4)$ & $0.9 \pm 1.4(0-5)$ & $<0.01$ \\
Paresthesia face & $0.1 \pm 0.6(0-4)$ & $0.6 \pm 1.2(0-5)$ & $<0.001$ \\
\hline
\end{tabular}


scores on the CINQ (59 vs. $17 ; p<0.001$ ) suggesting more severe patient-reported neuropathy symptoms.

The $\mathrm{CT}+$ group experienced significantly more paresthesias and dysesthesias in the upper $(p<0.001 ; p<0.01)$ and lower extremities $(p<0.001)$ than $\mathrm{CT}-$ patients (Table 2). In addition, the CINQ reveals that $\mathrm{CT}+$ patients had significantly more difficulty with fine motor function. They experienced more problems with buttoning up a blouse $(p<0.001)$, writing $(p<0.003)$, and picking up small objects $(p<0.01)$. They further reported more coldinduced pain when outside $(p<0.001)$ and when taking something out of the refrigerator $(p<0.001)$, more problems with the consumption of cold foods $(p<0.001)$ and drinks $(p<0.001)$ and more problems with controlling their bladder function $(p<0.05)$.

The CINQ further disclosed patients' problems with taking care of their environment due to neuropathies. Patients experienced limitations in their activities due to problems in hands, foot, or mouth $(p<0.001)$ and they mentioned difficulties with accepting the consequences of neurotoxicity $(p<0.001)$. Their neuropathies further limited them with gardening $(p<0.05)$ and sport activities $(p<0.01)$. Overall, patients indicated that neuropathy had a negative effect on QoL.

\section{Criterion validity of the CINQ}

Analyses showed a strong significant negative correlation between the FACT/GOG and the CINQ $(-0.73 ; p \leq 0.001)$ which suggests that severe patient-reported neuropathy symptoms were associated with a lower QoL. The Ntx and the CINQ scores were also strongly correlated negatively $(-0.84 ; \mathrm{p} \leq 0.001)$ which indicates that both questionnaires assess a similar construct. However, the current findings are preliminary and formal validation with clinical formal assessment of neuropathy is still needed.

\section{Discussion}

Our findings suggest that neurotoxicity has a considerable negative impact on daily activities and QoL in cancer patients treated with chemotherapy. In particular, paresthesias and dysesthesias in upper and lower extremities, problems with fine motor function, problems with controlling their bladder function, and cold-induced paresthesias were frequently reported. Most of these patients were treated with oxaliplatin, which is known for its cold-induced paresthesias $[6,8,10,15]$.

In previous studies, the influence of neurotoxicity on QoL has been evaluated with the FACT-Taxane [1], FACT/GOG-Ntx [2, 17] and the EORTC-QLQ-C30 [5, 7]. However, the specific effects of neurotoxicity on daily activities and the inconveniences for the patient do not become evident with these measures. Therefore, the CINQ was developed and used in this study. The CINQ is unique in revealing the problems in daily living caused by neurotoxicity. The strong association between the CINQ and FACT/GOG can be considered as preliminary support for its validity. With increasing neurotoxicity, a greater decrease in QoL can be expected.

The degree of neurotoxicity as assessed in the present study is hard to compare to other studies. There is currently no consensus about the gradation of neurotoxicity. The World Health Organization criteria [18], the Eastern Cooperative Oncology Group score [19], or the Ajani score [7] are sometimes used, while in other studies nerve conduction tests have been applied. In this study, no gradation has been made.

Although our results showed a strong negative association between FACT/GOG and CINQ, and although the FACT/GOG-Ntx subscale and CINQ scores were strongly associated, the CINQ needs to be further evaluated, in particular its reliability and validity. Also, the lack of an objective method of neurological assessment as a reference is a limitation. In addition, the relatively small number of patients did not allow us to investigate the influence of differential exposure to the various chemotherapeutic agents on patient-reported neuropathy symptoms. Future studies should investigate this further. Finally, the small number of included patients limits the generalizability of the results.

Despite these limitations, the present study is the first to provide support for the notion that neurotoxicity has a great impact on daily functioning of cancer patients and that these effects may require considerable adjustments. It is important for health care professionals to ask patients about problems related to neurotoxicity since the impact on daily activities may be considerable. Currently, hardly any treatment or preventive interventions are available $[6,8,9]$. However, patients should be adequately informed about possible side effects of their treatment. The CINQ might be a useful and promising measure to evaluate patients' suffering from neuropathy symptoms. However, validation against clinical formal assessment of neuropathy is still needed.

Acknowledgement The authors thank all patients for their participation in the study.

Conflict of interest None declared.

Open Access This article is distributed under the terms of the Creative Commons Attribution Noncommercial License which permits any noncommercial use, distribution, and reproduction in any medium, provided the original author(s) and source are credited. 


\section{References}

1. Markman M (1996) Chemotherapy-associated neurotoxicity: an important side-effect impacting on quality, rather than quantity of life. J Cancer Res Clin Oncol 122:511-512

2. Calhoun EA, Welshman EE, Chang CH, Lurain LR, Fishman DA, Hunt TL, Cella D (2003) Psychometric evaluation of the Functional Assessment of Cancer Therapy/Gynaecologic Oncologic GroupNeurotoxicity (FACT/GOG-Ntx) questionnaire for patients receiving systemic chemotherapy. Int J Gynecol Cancer 13:741-748

3. Kopec JA, Land SR, Cecchini RS, Ganz PA, Cella D, Costantino JP, Wieand S et al (2006) Validation of a self-reported neurotoxicity scale in patients with operable colon cancer receiving oxaliplatin. J Support Oncol 4:W1-W8

4. Almadrones L, Deborah B, Guire M, Walczak J, Colleen M, Tian C (2004) Psychometric evaluation of two scales assessing functional status and peripheral neuropathy associated with chemotherapy for ovarian cancer: a gynecologic oncology study group. Oncol Nurs Forum 31:615-623

5. Postma TJ, Heimans JJ, Aaronson NK, Grant R, Huddart R, Maher J, Hildehrand JG et al (1999) The impact of chemotherapy induced peripheral neuropathy on quality of life. J Neurol Neurosurg Psychiatry $67: 838$

6. Verstappen CP, Heimans JJ, Hoekman K (2003) Neurotoxic complications of chemotherapy in patients with cancer: clinical signs and optimal management. Drugs 63:1549-1563

7. Postma TJ, Heimans JJ (2000) Grading of chemotherapy-induced peripheral neuropathy. Ann Oncol 11:509-513

8. Quasthoff S, Hartung HP (2002) Chemotherapy-induced peripheral neuropathy. J Neurol 249:9-17

9. Visovsky C (2003) Chemotherapy-induced peripheral neuropathy. Cancer Invest 21:439-451
10. Grothey A (2005) Clinical management of oxaliplatin-associated neurotoxicity. Clin Colorect Cancer 5:38-46

11. Durand JP, Brezault C, Goldwasser F (2003) Protection against oxaliplatin acute neurosensory toxicity by venlafaxine (effexor). Anti-Cancer Drugs 14:423-425

12. Gamelin L, Bisdron-Celle M, Delva R, Guerin-Meyer V, Ifrah N, Morel A, Gamelin E (2004) Prevention of oxaliplatin-related neurotoxicity by calcium and magnesium infusions: a retrospective study of 161 patients receiving oxaliplatin combined with 5Fluoroucil and Leucovorin for advanced colorectal cancer. Clin Cancer Res 10:4055-4061

13. Mielke S, Mross K, Gerds TA, Schmitdt A, Wasch R, Berger DP, Lange W et al (2003) Comparative neurotoxicity of weekly non-break paclitaxel infusions over 1 versus $3 \mathrm{~h}$. Anti Cancer Drugs 14:785-792

14. Screnci D, Mc Keage MJ (1999) Platinum neurotoxicity: clinical profiles, experimental models and neuroprotectieve approaches. J Inorg Biochem 77:105-110

15. Gamelin E, Gamelin L, Bossi L, Quasthoff S (2002) Clinical aspects and molecular basis of oxaliplatin neurotoxicity: current management and development of preventive measures. Seminars Oncol 29:21-33

16. Leonard GD, Wright MA, Quinn MG (2005) Survey of oxaliplatin-associated neurotoxicity using an interview-based questionnaire in patients with metastatic colorectal cancer. BMC Cancer 5:116-125

17. Huang HQ, Brady MF, Cella D, Fleming G (2007) Validation and reduction of FACT/GOG-Ntx subscale for platinum/paclitaxel-induced neurologic symptoms: a gynecologic oncology group study. Int $\mathrm{J}$ Gynecol Cancer 17:387-393

18. Miller AB, Hoogstraten B, Staquet M, Winkler A (1981) Reporting results of cancer treatment. Cancer 47:207-214

19. Oken MM (1982) Toxicity and response criteria of the Eastern Cooperative Oncology Group. Am J Clin Oncol 5:649-655 